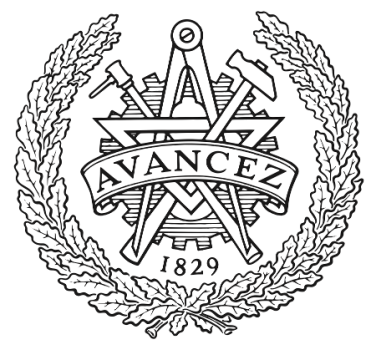

CHALMERS

UNIVERSITY OF TECHNOLOGY

\title{
The Molecular Fingerprint of Fluorescent Natural Organic Matter Offers Insight into Biogeochemical Sources and Diagenetic State
}

Downloaded from: https://research.chalmers.se, 2023-04-26 12:47 UTC

Citation for the original published paper (version of record):

Wuensch, U., Acar, E., Koch, B. et al (2018). The Molecular Fingerprint of Fluorescent Natural Organic Matter Offers Insight into

Biogeochemical Sources and Diagenetic State. Analytical Chemistry, 90(24): 14188-14198.

http://dx.doi.org/10.1021/acs.analchem.8b02863

N.B. When citing this work, cite the original published paper. 


\title{
The Molecular Fingerprint of Fluorescent Natural Organic Matter Offers Insight into Biogeochemical Sources and Diagenetic State
}

\author{
Urban J. Wünsch, ${ }^{*},+\ddagger$ Evrim Acar, ${ }^{\S}$ Boris P. Koch, ${ }^{\|, \perp}$ Kathleen R. Murphy, ${ }^{\dagger}$ \\ Philippe Schmitt-Kopplin, ${ }^{\#}$ and Colin A. Stedmon
}

${ }^{\dagger}$ Chalmers University of Technology, Architecture and Civil Engineering, Water Environment Technology, Sven Hultins Gata 6, 41296 Gothenburg, Sweden

${ }^{\ddagger}$ National Institute of Aquatic Resources, Technical University of Denmark, Kemitorvet, 2800 Kgs. Lyngby, Denmark

${ }^{\S}$ Simula Metropolitan Center for Digital Engineering, Pilestredet 52, 0167 Oslo, Norway

"Alfred Wegener Institute Helmholtz Centre for Polar and Marine Research, Am Handelshafen 12, 27570 Bremerhaven, Germany

${ }^{\perp}$ University of Applied Sciences, An der Karlstadt 8, 27568 Bremerhaven, Germany

${ }^{\#}$ Research Unit Analytical Biogeochemistry (BGC), Helmholtz Zentrum München, German Research Center for Environmental Health, Ingolstädter Landstrasse 1, 85764 Neuherberg, Germany

\section{Supporting Information}

\begin{abstract}
Investigating the biogeochemistry of dissolved organic matter (DOM) requires the synthesis of data from several complementary analytical techniques. The traditional approach to data synthesis is to search for correlations between measurements made on the same sample using different instruments. In contrast, data fusion simultaneously decomposes data from multiple instruments into the underlying shared and unshared components. Here, Advanced Coupled Matrix and Tensor Factorization (ACMTF) was used to identify the molecular fingerprint of DOM fluorescence fractions in Arctic fjords. ACMTF explained 99.84\% of the variability with six fully shared components. Individual molecular formulas were linked to multiple fluorescence
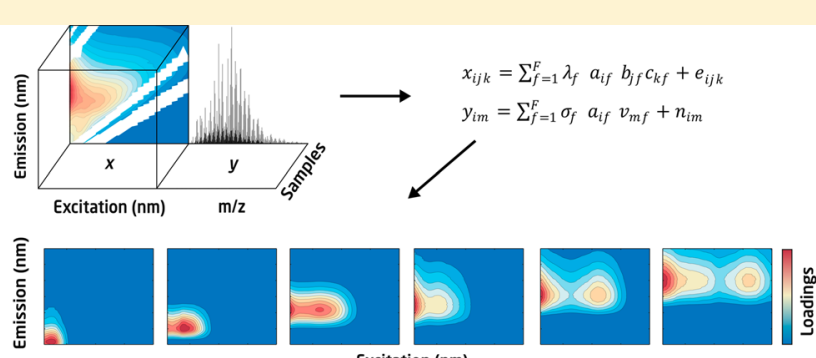

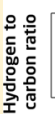
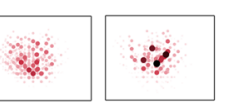

Excitation (nm)
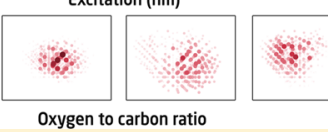

Oxygen to carbon ratio components and vice versa. Molecular fingerprints differed in diversity and oceanographic patterns, suggesting a link to the biogeochemical sources and diagenetic state of DOM. The fingerprints obtained through ACMTF were more specific compared to traditional correlation analysis and yielded greater compositional insight. Multivariate data fusion aligns extremely complex, heterogeneous DOM data sets and thus facilitates a more holistic understanding of DOM biogeochemistry.
\end{abstract}

$\mathrm{T}$ he complex multifaceted interactions between dissolved organic matter (DOM) and biological ${ }^{1}$ and physical processes $^{2}$ cements its central role in aquatic ecosystems. ${ }^{3}$ The wide variety of environmental processes involved results in an extremely complex pool of organic compounds that spans nearly all possibilities defined by the laws of chemical bonding. ${ }^{4-6}$ In order to understand the biogeochemical role of DOM in natural waters, it is essential to reduce the complexity of analytical data and to trace and characterize various underlying fractions. Owing to its molecular complexity, the simultaneous quantification and characterization of DOM presents a formidable analytical challenge. Users often have to choose between techniques with different strengths and limitations. ${ }^{7,8}$ The insight gained from independent analytical techniques strongly depends on the experimental design and data analysis approach and is ultimately limited by the intrinsic constraints of the individual approach.

The determination of ultraviolet-visible (UV-vis) spectroscopic properties (targeting chromophoric and fluorescent
DOM, CDOM, and FDOM, respectively) represents a rapid method to follow DOM dynamics. ${ }^{9,10}$ An ever-increasing number of studies focus on FDOM, since its measurement is cost-efficient, highly sensitive, and suitable for field deployment. ${ }^{11}$ Fluorescence excitation emission matrices (EEMs) are frequently decomposed into the underlying independently fluorescing components using multiway techniques such as Parallel Factor Analysis (PARAFAC). ${ }^{12}$ However, since fluorescence and absorbance require optically active compounds, they can only target a fraction of the DOM pool. The chemical structures responsible for the UV-vis spectroscopic properties of DOM have yet to be uncovered. ${ }^{10}$ Moreover, the chemical interpretation of PARAFAC spectra is inherently difficult and often results in the ambiguous labeling of

Received: June 25, 2018

Accepted: November 13, 2018

Published: November 18, 2018 
components that suggests representation of molecular species (e.g., proteins or humic substances). ${ }^{13}$

Another analytical approach to DOM characterization is ultrahigh-resolution mass spectrometry, ${ }^{14}$ which determines the exact masses, and thus molecular formulas, of organic substances present. ${ }^{5}$ Importantly, while DOM can be massresolved, ultrahigh-resolution mass spectrometry cannot routinely distinguish structural isomers for a given mass peak and thus still produces convoluted analytical signals that can be challenging to interpret in complex mixtures. To date, this has mainly been addressed by multivariate analysis with Principal Component Analysis (PCA) or Hierarchical Cluster Analysis. ${ }^{15}$ PCA in particular is a powerful approach to reduce complexity and isolate factor loadings that correspond to the chemical imprint of environmental processes. However, the properties of the decomposition might hinder the discovery of true chemical signals. For example, in PCA, components are orthogonal; i.e., all factors have a loading similarity of zero. However, many properties of DOM can be expected to be correlated (nonorthogonal). A more flexible multivariate approach to distinguish underlying factors of molecular formula matrices is therefore needed.

The comparison of fluorescence spectroscopy and ultrahighresolution mass spectrometry makes apparent that the strengths and limitations of both approaches are diametrically opposite: The former approach, when coupled with PARAFAC, allows meaningful statistical description but offers only limited insight into DOM chemical composition, while the latter offers a wealth of qualitative chemical information with limited means to systematically elucidate the primary factors responsible for observed dynamics. Experience in other disciplines, such as metabolomics, shows that considerable analytical advances are achieved when two or more complementary data sets are jointly analyzed. ${ }^{16}$ For DOM, recent studies have employed post hoc rank-correlation analysis to establish links between optical and chemical properties of DOM. ${ }^{17,18}$ However, considering that thousands of signals are compared, the risk of false positive correlations is significant. Moreover, correlations may sometimes be hard to interpret, e.g., when negative correlations are reported while both signals in principle correspond to analyte concentrations. A promising approach is to jointly decompose these data sets into multiple underlying factors using advanced data fusion that can account for their convoluted character. However, no such models have been tested for DOM.

The heterogeneous nature of DOM data sets requires an approach that can handle the different types of data sets while accounting for the partial overlap of detector signals. A data fusion model based on simultaneous factorization of multiple data sets, called Advanced Coupled Matrix and Tensor Factorization (ACMTF), has been developed specifically for such scenarios. ${ }^{19}$ In recent years, ACMTF has been used in metabolomics ${ }^{20}$ and medical applications. ${ }^{21}$ Here, we applied ACMTF to simultaneously analyze and decompose data from two popular DOM characterization techniques, fluorescence EEMs and Fourier transform ultrahigh-resolution mass spectrometry (FT-ICR-MS) molecular formulas $(N=527)$ for samples $(N=174)$ originating from three Arctic Fjords. The associations reported by the statistical components identified by ACMTF are subsequently compared to those suggested by post hoc correlation analysis. We propose that data fusion represents a vital step toward a more holistic data analysis that can help to better elucidate the complex dynamics of DOM.

\section{MATERIALS AND METHODS}

Sample Collection. In July 2016, 174 water samples were collected onboard R/V Maria S. Merian (cruise MSM56, Ecological Chemistry in Arctic Fjords) over a three-week period (see Figure $\mathrm{S} 1$ and Table $\mathrm{S} 1$ for an overview of sampling regions, salinity, temperature, DOC, and depth ranges). The transect encompassed three fjords (Kongsfjorden [Longyearbyen], Scoresby Sound [East Greenland], and Arnarfjördur [West Iceland]) spanning from $79^{\circ} \mathrm{N}$ to $65^{\circ} \mathrm{N}$ and $28^{\circ} \mathrm{W}$ to $12{ }^{\circ} \mathrm{E}$. Kongsfjorden and Scoresby Sound both have marine terminating glaciers, while Arnarfjördur further south in Iceland does not. All fjords receive limited DOM input from rivers in their catchments, and the flux of marine DOM from the shelf and production of DOM associated with plankton productivity dominate. Scoresby Sound, similar to many East Greenland fjords, receives terrestrial organic matter from the Arctic transported in the East Greenland Current (EGC) and has low productivity. Kongsfjorden and Arnarfjördur are not influenced by the EGC and are primarily supplied with Atlantic water with little or no terrestrial DOM. Water samples were collected at depths ranging from 1.7 to $1397 \mathrm{~m}$ with a 24bottle CTD Rosette, equipped with Niskin bottles and immediately filtered using precombusted GF/F filters (0.7 $\mu \mathrm{m}$, Whatman) by applying a vacuum of $<200$ mbar. After filtration, DOM was immediately solid-phase extracted using $200 \mathrm{mg}$ of PPL resins as described previously. ${ }^{22}$ Cartridges were desalted and dried onboard and stored dark and frozen at $-20{ }^{\circ} \mathrm{C}$. In the home laboratory, DOM was eluted with 1000 $\mu \mathrm{L}$ of methanol. The final extract volume was determined by weight, and samples were stored at $-18{ }^{\circ} \mathrm{C}$ until analysis.

Spectroscopic Measurements. A total of $50 \mu \mathrm{L}$ of DOM extract (in methanol) was dried using a gentle stream of $\mathrm{N}_{2}$ at room temperature, reconstituted in $4 \mathrm{~mL}$ of $150 \mathrm{mM}$ ammonium acetate $(\mathrm{pH} \mathrm{7)}$ in precombusted amber glass vials, and equilibrated at room temperature for $30 \mathrm{~min}$. Fluorescence and absorbance measurements were obtained using a HORIBA AquaLog fluorometer using a $10 \mathrm{~mm}$ quartz cuvette (Helma Analytics). Fluorescence emission was detected in the range of $240-600 \mathrm{~nm}$ (increment $\sim 3.3 \mathrm{~nm}$ ) at excitation wavelengths between 240 and $450 \mathrm{~nm}$ (increment $3 \mathrm{~nm}$ between 240 and $360 \mathrm{~nm}, 9 \mathrm{~nm}$ between 360 and 450 $\mathrm{nm})$. A separate absorbance measurement was carried out to measure absorbance between 240 and $600 \mathrm{~nm}$ at increments matching the fluorescence excitation. The fluorescence data were processed using the $d r E E M$ toolbox. ${ }^{12}$ Data were corrected for inner filter effects using the absorbance-based method (absorbance between 0.014 and $0.07 \mathrm{~cm}^{-1}$ at 260 $\mathrm{nm}){ }^{23}$ First and second order physical scatter was removed and not interpolated. A fluorescence EEM of $150 \mathrm{mM}$ ammonium acetate was subtracted as the spectroscopic blank. Average EEMs for the three fjords are depicted in Figure S2.

Spectrometric Measurements. Fourier transform ion cyclotron resonance mass spectra were collected with a $12 \mathrm{~T}$ Bruker Solarix mass spectrometer (Bruker Daltonics, Bremen, Germany) using an Apollo II electrospray ionization (ESI) source in negative ionization mode. Samples were diluted in $50 / 50(\mathrm{v} / \mathrm{v})$ methanol/water to a concentration of $1.5 \mathrm{nmol}$ DOC $\mathrm{mL}^{-1}$ and injected into an electrospray source at a flow rate of $0.12 \mathrm{~mL} \mathrm{~h}^{-1}$ with a nebulizer gas pressure of $2.2 \mathrm{bar}$ and 
a drying gas flow rate of $4 \mathrm{~L} \mathrm{~min}^{-1}$. Spectra were externally calibrated using arginine clusters and then internally calibrated using marine DOM molecular formulas. ${ }^{24}$ The spectra were acquired at 4 Mega words over a mass range of $m / z 100-1000$, and 300 scans were accumulated for each spectrum. The average mass resolution of all signals at $400 \mathrm{~m} / z$ was 375000 . The formula assignment was carried out as detailed in the SI. Briefly, molecular formulas were calculated from $\mathrm{m} / z$ values allowing for elemental combinations ${ }^{12} \mathrm{C}_{0-\infty},{ }^{13} \mathrm{C}_{0-1},{ }^{1} \mathrm{H}_{0-\infty}$, ${ }^{14} \mathrm{~N}_{0-2},{ }^{16} \mathrm{O}_{0-\infty}$, and ${ }^{32} \mathrm{~S}_{0-1}$; a mass accuracy threshold of $|\Delta m|$ $\leq 0.2 \mathrm{ppm}$; and a relative abundance of $>1 \%$. Formulas which were either detected in process blanks (PPL extraction of ultrapure water) or contained in the list of potential surfactants were removed from the entire data set. $^{25}$ It should be considered that every assigned molecular formula most likely implies an immense structural diversity of isomers. ${ }^{6}$ Since FTICR-MS alone is unable to distinguish between such structural isomers, we reserve the term component for statistical components and use the term molecular formula in the context of spectrometric signals.

Data Processing. In order to describe optical and chemical properties of DOM using statistical models, data were preprocessed as follows: (1) EEM data were normalized to reduce concentration effects and match the character of relative formula abundances (using the "normeem" function in drEEM). (2) Signals in EEMs and the molecular formula matrix were scaled with their Euclidean norm to equalize their numerical leverage. (3) FT-ICR-MS formula abundances were scaled by division with the square root of the standard deviation to reduce modeling leverage of highly abundant formulas. (4) Undetected FT-ICR-MS formulas in a given sample were assigned as "missing," and formulas with more than $3.6 \%$ missing detections (i.e., more than 10 samples without formula observation) were excluded from further analysis ( $81 \%$ of the 2776 formulas in the original data set). While this represents a significant reduction in molecular formula data, a linkage between fluorescence signals present in all samples with molecular formulas only present in a small fraction of samples is unlikely. Preliminary ACMTF models indicated high residuals below $m / z 300$ and above $m / z 500$, therefore these formulas were removed to mitigate disturbances. The resulting data sets had the following structure and dimensions: sample $\times$ emission $\times$ excitation $(174 \times 91 \times 44)$ and sample $\times$ formula abundance $(174 \times 527)$ for fluorescence EEMs and FT-ICR-MS formula abundances, respectively. On the basis of the original relative molecular formula abundances, the subset of 527 modeled molecular formulas represented 38 $\pm 5 \%$ of the ESI-MS molecular formula abundance (Figure S3).

Advanced Coupled Matrix and Tensor Factorization. A detailed description of the fundamental principle of the ACMTF model is presented in the SI (section S1). Briefly, ACMTF jointly decomposes fluorescence EEMs and molecular formula matrices into a set of trilinear fluorescence components and bilinear molecular formula components by fitting a PARAFAC model to the fluorescence EEMs and factorizing the molecular formula matrix in a way that the component scores are identical. Component weights ( $\lambda$ for EEMs and $\sigma$ for formula matrices) are used to evaluate whether a particular component is shared between both analytical data sets. ACMTF modeling was carried out using the Matlab CMTF toolbox ${ }^{19,26}$ in conjunction with the Tensor toolbox $^{27}$ and the SNOPT toolbox. ${ }^{28}$ ACMTF factorization is computationally intense, and calculations were therefore carried out using a set of IBM NeXtScale nx360 M4 nodes, with 100 models being fit simultaneously (reducing the analysis time for 100 models from 6 days on a single-core computer to $3 \mathrm{~h}$ with parallel computing).

Nonnegativity constraints in all modes of both data sets were applied during the modeling. Furthermore, angular constraints in the excitation mode were applied to prevent the algorithm from converging on solutions with highly similar factors (violating model assumptions): Model components were constrained to have Tucker Congruence Coefficient (TCC) values between all excitation spectra of less than 0.93 (limit set by maximum similarity between PARAFAC excitation spectra). ACMTF models were evaluated by (1) an assessment of the fluorescence spectra (chemical coherence), (2) a variability assessment of the component weights $\lambda$ and $\sigma$ (model uniqueness), and (3) split-half validation. ACMTF component scores were converted to $F_{\max }$ values by multiplying component scores with the spectral maximum of fluorescence excitation and emission, which returns scores in the unit of the modeled data. Here, $F_{\max }$ values represent unitless, relative values, since both data sets were scaled and normalized prior to analysis. Contrary to similar procedures during PARAFAC analysis, these preprocessing steps are currently irreversible.

The chemodiversity of ACMTF components was estimated as the richness estimator Chao 1 using the $\mathrm{R}$ software package vegan ( $\mathrm{R}$ v3.5.1) with molecular formulas as species and component loadings as species counts. ${ }^{29,30}$ To mimic species counts, the loadings of all components were normalized by the maximum loading across all components, multiplied by 100 , and rounded in order to represent integer species counts.

Parallel Factor Analysis, Principal Component Analysis, and Pearson Rank Correlation. The underlying components of fluorescent DOM in 191 samples (data set contained a small number of samples for which no mass spectra were collected) were isolated using Parallel Factor Analysis using the $d r E E M$ toolbox. ${ }^{12}$ To do so, models with different numbers of components with nonnegative loadings and scores were explored (four to seven components). Ultimately, a six-component PARAFAC model with a core consistency of $1.5 \%$ and an explained variance of $99.9 \%$ was found to best represent the data set. ${ }^{31}$ This model was validated using a split-half validation, for which the whole data set $(N=191)$ was split into six separate randomly split halves $(94>N<97)$.

PCA was performed on the molecular formula matrix exclusively to determine the explanatory power of this technique in comparison to ACMTF and to compare the extent of autocorrelation between component loadings. A six component PCA model was calculated for autoscaled and mean-centered molecular formula abundances (in addition to the preprocessing detailed above). PCA models were calculated using PLS toolbox in Matlab (Eigenvector Research Inc. v.8.52). Factor similarities between ACMTF and PCA components were quantified using Tucker congruence coefficients for all unique combinations of components $(N=$ $15)^{32}$

Pearson rank correlation was performed on the processed data set described above. Molecular formula relative abundances were correlated to the $F_{\max }$ values of a split-half validated six component PARAFAC model using only pairwise complete comparisons. A Holm-Bonferroni correction for multiple comparisons was applied to address the possibility of 

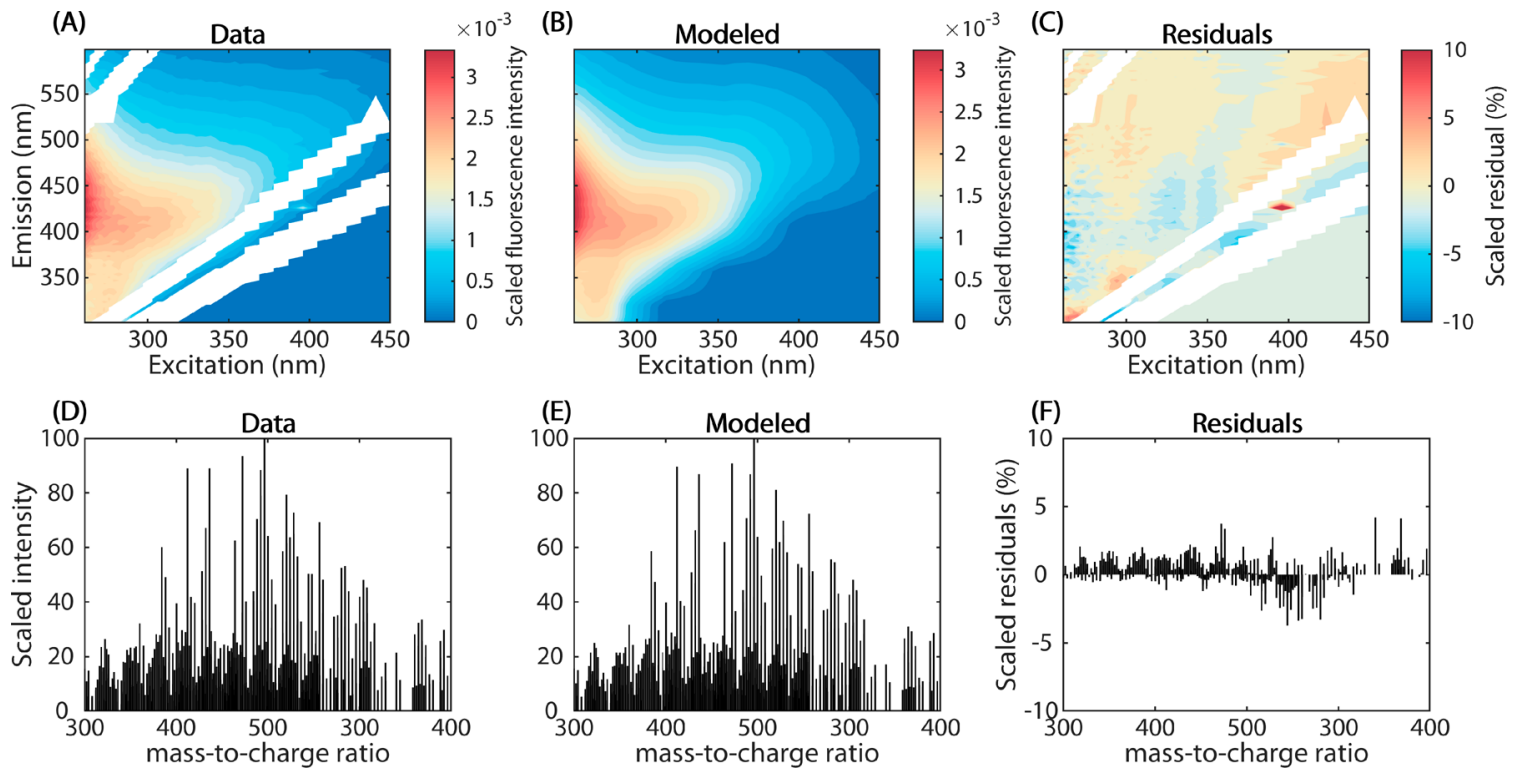

Figure 1. Example of measured vs modeled data. (A-C) Fluorescence EEMs (scaled and therefore unitless intensities). (D-F) FT-ICR-MS mass spectra scaled by the maximum peak intensity. First column: Examples of raw data. Second column: Examples of corresponding modeled data. Third column: Model residuals, scaled by the maximum peak intensity in the sample. The depicted sample was taken in Kongsfjorden, Norwegian monitoring station $\mathrm{Kb} 5\left(78.9^{\circ} \mathrm{N}, 12.4^{\circ} \mathrm{E}\right)$ at a depth of $30 \mathrm{~m}$.
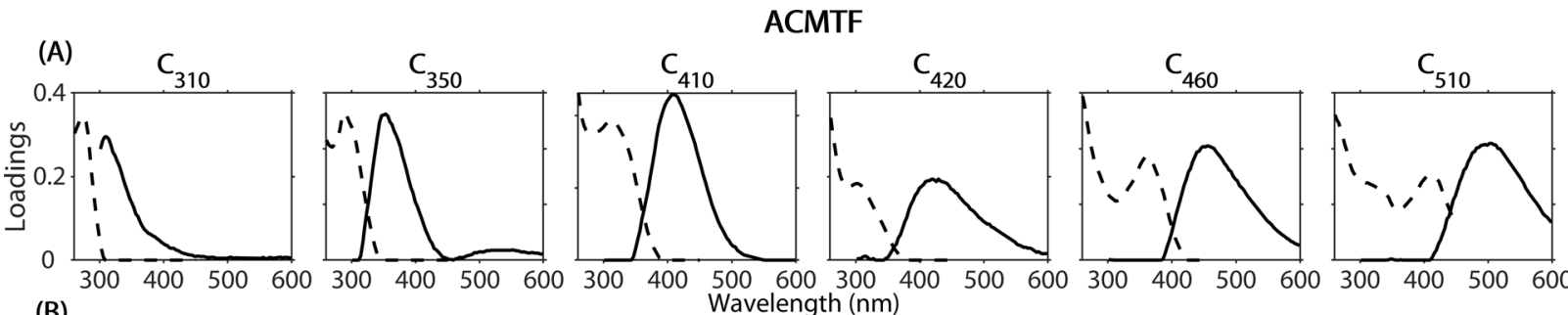

(B)
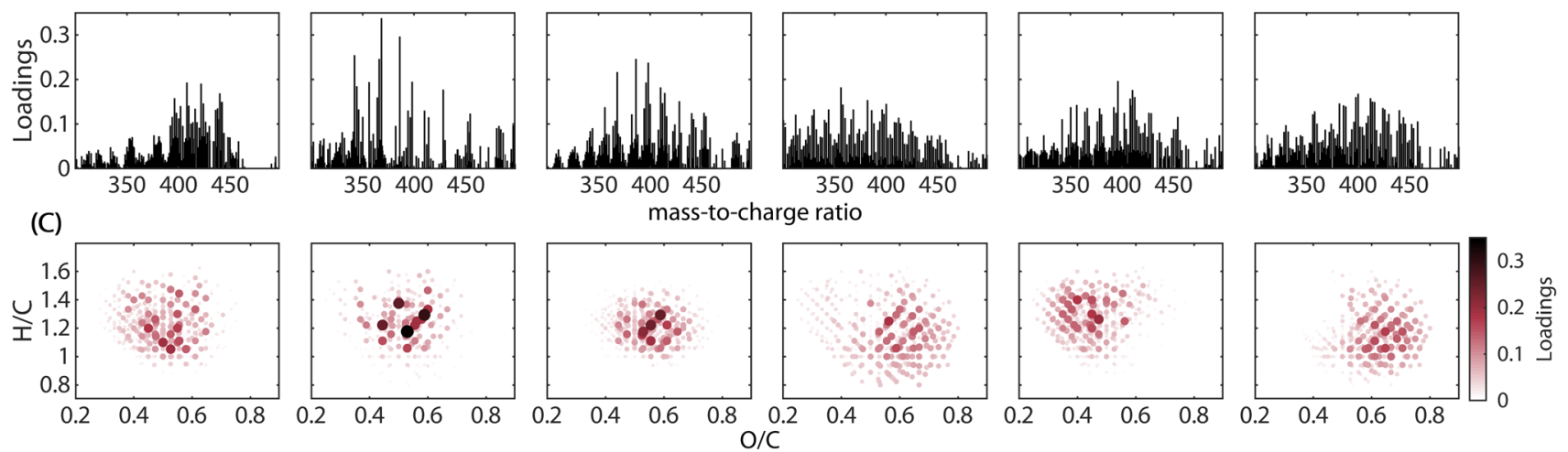

(D)
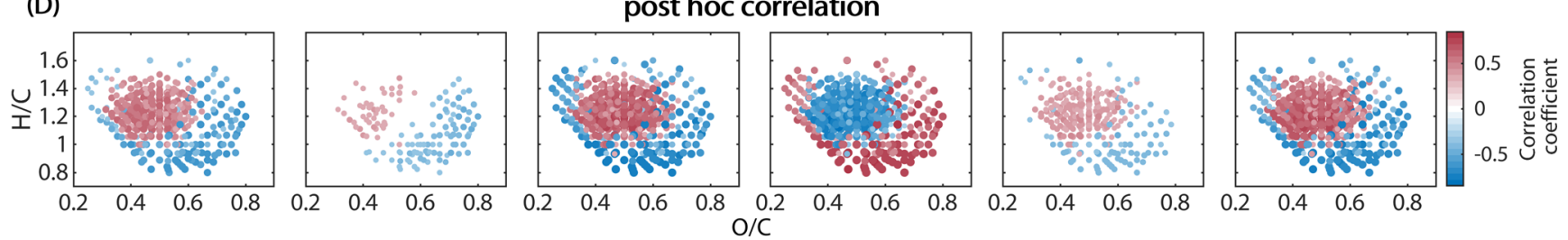

Figure 2. The molecular fingerprint of fluorescent DOM as identified by ACMTF and post hoc correlation. Panel A depicts fluorescence loadings as a function of excitation (dashed line) and emission (solid line); panel B and C show molecular formula loadings as a function of mass-to-charge ratio and molecular composition in the van Krevelen space. For comparison, the correlation between PARAFAC component scores (spectrally congruent with components in the top row, also provided in Figure S8) is shown in panel D. Linear correlation coefficients were restricted to $p<$ 0.01 prior to visualization and are corrected for false-positives using a Holm-Bonferroni correction.

type I errors, ${ }^{33}$ eliminating $19.5 \%$ of correlations that would have otherwise been reported as significant. The matrix of correlation coefficients $(r)$ was subsequently restricted to comparisons satisfying the significance threshold of $\alpha=0.01$; 
Table 1. Properties of ACMTF Components ${ }^{a}$

\begin{tabular}{|c|c|c|}
\hline component & $\lambda$ (EEMs) & $\sigma(\mathrm{FT}-\mathrm{ICR}-\mathrm{MS})$ \\
\hline $\mathrm{C}_{310}$ & $0.22 \pm 0.005$ & $0.12 \pm 0.002$ \\
\hline $\mathrm{C}_{350}$ & $0.24 \pm 0.003$ & $0.13 \pm 0.007$ \\
\hline $\mathrm{C}_{410}$ & $0.46 \pm 0.007$ & $0.40 \pm 0.005$ \\
\hline $\mathrm{C}_{420}$ & $0.15 \pm 0.002$ & $0.15 \pm 0.002$ \\
\hline $\mathrm{C}_{460}$ & $0.27 \pm 0.003$ & $0.32 \pm 0.004$ \\
\hline $\mathrm{C}_{510}$ & $0.19 \pm 0.002$ & $0.17 \pm 0.003$ \\
\hline
\end{tabular}

${ }^{a}$ Component weights $(\lambda$ and $\sigma)$ indicate the contribution of a component to the overall variability and indicate their sharedness across EEMs and FT-ICR-MS. Weighted averages (subscript wa) refer to the weighted average chemical composition of specific components. O/C: Oxygen-tocarbon ratio. H/C: Hydrogen-to-carbon ratio. m/z: mass-to-charge ratio. DBE: Double bond equivalent. N/C: Nitrogen-to-carbon-ratio. Ci: Chemodiversity index.

correlation coefficients with $p>\alpha$ (based on HolmBonferroni corrected $\mathrm{p}$ values) were ignored in subsequent analyses.

\section{RESULTS AND DISCUSSION}

Model Validation. Similar to other multivariate models such as PARAFAC, the validity of ACMTF models primarily depends upon the applicability of the underlying model to explain the data set variability, as well as choosing the right number of components. The application of data fusion furthermore depends on a stable, reproducible relationship between signals obtained on different instruments. ACMTF was applied under the assumption that the statistically identifiable signals in fluorescence EEMs and molecular formulas respond linearly to the presence of the corresponding (unknown) analytes. The adherence to this assumption was investigated by judging the robustness and representativeness of the model. This validation of the selected ACMTF model was carried out by analyzing the overall degree of explained variance, the randomness of residuals, the chemical coherence of component loadings, and the ability of reproducing the overall model from fully independent subsets of the overall data set.

After the initial data exploration, a six-component ACMTF model was found to best explain the variability in fluorescence EEMs and the molecular formula matrix. With six components, ACMTF explained $99.84 \%$ of variance in both data sets and featured mostly random, low model residuals (example shown in Figure 1, Figure S4). The properties of fluorescence spectra (Figure 2, top row) were generally consistent with those observed for pure fluorophores (single emission peak, Stokes' shift between 0.55 and $1.13 \mathrm{eV}$, Table 1). Further investigations, described in the Supporting Information (SI, Figures S4-S8, Table S2), indicated the suitability of ACMTF for the simultaneous decomposition of fluorescence EEMs and molecular formulas. The split-half validation indicated that a relatively low number of the modeled formulas did not produce the same component loadings in both independent data set halves (Figure S6). This indicates that the dynamics of this small subset of modeled formulas deviated from the ideal, linear behavior to some degree. It is possible that these molecular formulas either were not detected reproducibly by ESI-MS or they represented independent molecular structures that were not represented by a statistical component (molecular fingerprint) present across a range of samples. Overall, the majority of analytical signals were represented by the ACMTF model in a robust fashion, and our results thus indicate that the analytical signals identified by ACMTF scaled linearly with analyte abundance.
Chemical Properties of Fluorescence Spectra. The sixcomponent ACMTF model featured fluorescence components with emission maxima at 310, 350, 410, 420, 460, and $510 \mathrm{~nm}$ (Figure 2, panel A, henceforth referred to by these emission maxima) and molecular formula components with distinctly different molecular weight distributions, elemental composition, and overall varying degrees of chemodiversity (Figure 2, panel B and C, Table 1). A comparison with the OpenFluor database $^{34}$ revealed similarities of all ACMTF fluorescence components with previously identified PARAFAC components $\left(\mathrm{TCC}_{\mathrm{ex}, \mathrm{em}}>0.98\right)$. Specifically, $\mathrm{C}_{310}, \mathrm{C}_{350}$, and $\mathrm{C}_{510}$ were similar to components identified in the coastal Canadian Arctic. ${ }^{35}$ However, matches were also observed across other aquatic environments, such as the Baltic Sea $\left(C_{310}\right.$ with $C 5$ in Stedmon et al.), small streams $\left(\mathrm{C}_{350}\right.$ with $\mathrm{C5}$ in Yamashita et al. or $\mathrm{C}_{410}$ with $\mathrm{C} 1$ in Graeber et al. $)$, or drinking water $\left(\mathrm{C}_{420}\right.$ with $\mathrm{C} 3$ in Shutova et al.). ${ }^{36-39}$

The component weights $\lambda$ and $\sigma$ (see SI section S1 for further details) indicated that components were generally shared between both data sets. For $\mathrm{C}_{410}, \mathrm{C}_{420}, \mathrm{C}_{460}$, and $\mathrm{C}_{510}, \lambda$ (weights for fluorescence components) and $\sigma$ (weights for molecular formula components) deviated less than $15 \%$ between each other, indicating shared components (Table 1). In contrast, weights differed by almost $80 \%$ for $C_{310}$ and $\mathrm{C}_{350}$. While this represents a significant difference, factors other than unshared signals may have contributed to this observation. In addition to "sharedness" of components in both data sets, weights in the ACMTF model also reflect contributions of a given component to the overall variability in each data set. The weights $\lambda$ and $\sigma$ are thus influenced by detector response. Furthermore, differences in component complexity may lead to different weights of shared components in separate data sets. In our application, the ionization efficiencies of molecular formulas associated with $\mathrm{C}_{310}$ and $\mathrm{C}_{350}$ compared to their fluorescence quantum yields may have been partly responsible for different component weights. However, further investigations are necessary to investigate this hypothesis and the response of component weights to analytical factors. Overall, despite $\mathrm{C}_{310}$ and $\mathrm{C}_{330}$ 's different component weights, these findings indicate that all modeled components were shared between both data sets and therefore represented interpretable, chemically meaningful components that allow the investigation of molecular formulas associated with fluorescence spectra.

ACMTF addresses the multivariate character of molecular formulas by dissecting the abundance of one formula into multiple components (Figure S9), and the identification of the most prominent links between formula and fluorescence described in a specific component is therefore complicated. 

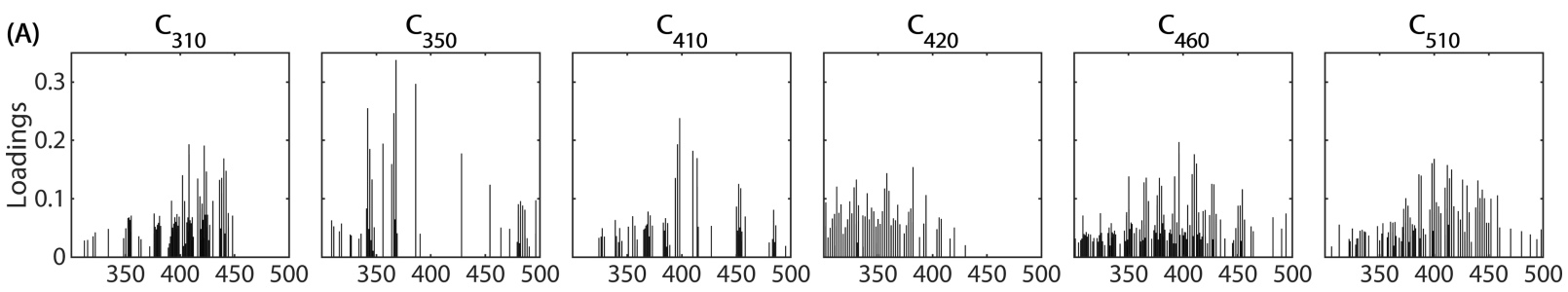

(B)
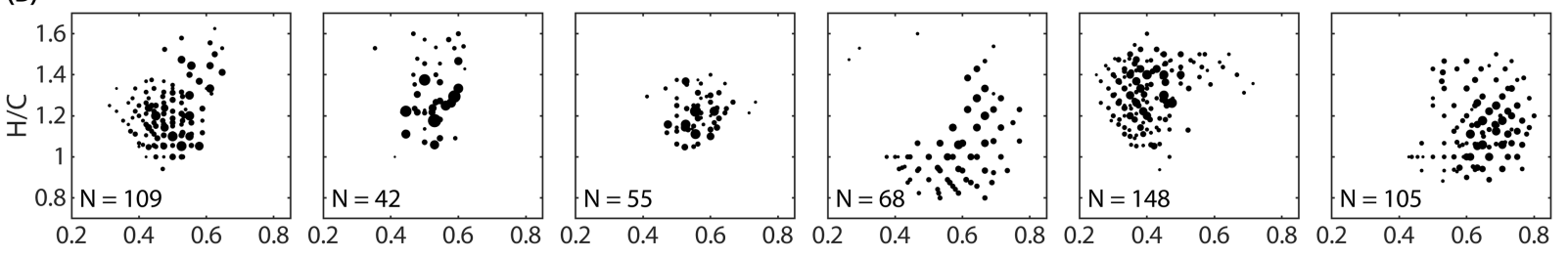

$\mathrm{O} / \mathrm{C}$

Figure 3. Modified component mass spectra of ACMTF components. For the purpose of simplification, every formula is only represented when it dominates the loading of a particular component (i.e., has the highest loading). Panel A: Modified component molecular weight distributions. Panel B: Modified component van Krevelen plots, where the size of dots represents the component loadings.

Here, we propose an approach to simplify the interpretation of ACMTF mass spectra for the purpose of initial investigations: The molecular formula loadings (Figure 2B,C) can be simplified by identifying the component with the highest relative loading for every molecular formula while disregarding the remaining components. In the resulting modified component mass spectra, every molecular formula is only represented once, and the interpretation is simplified (Figure S9 depicts several examples).

When plotting modified mass spectra in the van Krevelen space (Figure 3), the chemical properties associated with fluorescence spectra clustered in specific regions. The modified component loadings tracked a continuum of chemical properties along a diagonal line in the van Krevelen space from the most saturated formulas (high $\mathrm{H} / \mathrm{C}$, low $\mathrm{O} / \mathrm{C}$ ) to the most oxygenated, unsaturated formulas (high $\mathrm{O} / \mathrm{C}$, low $\mathrm{H} / \mathrm{C}$ ) where oxygenation and unsaturation increased in the order $\mathrm{C}_{460}<\mathrm{C}_{310}<\mathrm{C}_{350}<\mathrm{C}_{410}<\mathrm{C}_{420}<\mathrm{C}_{510}$. This shift of elemental composition between components observed in the modified component spectra accurately reflected the properties of the unmodified components revealing identical shifts in the weighted averages of $\mathrm{O} / \mathrm{C}$ and $\mathrm{H} / \mathrm{C}$ (Table 1 ). This indicates that the shift is an inherent pattern and not an artifact of the simplification of the molecular fingerprint of each component to modified loadings. However, as would be expected, there was a large discrepancy between the chemodiversity of molecular formula components (Table 1) and the number of formulas represented in modified mass spectra $(148>N>42$, Figure 3). Despite considerable data reduction, modified mass spectra appear to offer adequate insight into the compositional differences between components, but further in-depth investigations of chemical properties require the consideration of the complete component molecular fingerprint and thus all further model interpretations refer to the loadings depicted in Figure 2C.

Because ACMTF indicated that all identified fluorescence components were linked (i.e., shared) with distinctly different mass spectra, our study allowed the first multivariate estimate of the molecular fingerprint of fluorescent organic matter. As can be seen in Figure 2C, the chemodiversity and elemental composition of components differed significantly, but fluorescence properties such as emission maximum or Stokes shift did not correlate with molecular properties such as chemodiversity, weighted average elemental composition, or weighted average molecular weight. This suggests that while EEM fluorescence was possibly caused by the excitation of one or several chemical moieties summarized in the molecular fingerprint of ACMTF components, these fluorescing moieties do not dominate the molecular fingerprint to an extent that would allow the usage of fluorescence properties as indicators for, or predictors of, associated molecular fractions (and vice versa). Moreover, it should not be assumed that the molecular formulas summarized in ACMTF components predominantly consist of moieties that fluoresce but rather that their dynamics are indistinguishable from the moieties that do. However, if further studies should reveal consistent trends in the molecular fingerprint of fluorescent DOM, the stable, albeit potentially noncausal relationship could be utilized routinely to expand the analytical window of UV-vis spectroscopic analyses.

Comparison of Data Fusion and Traditional Approaches. Since the present study is the first application of ACMTF to analytical DOM data sets, it is important to compare the results obtained with ACMTF to existing approaches employed in previous studies. First, the factorization of molecular formulas with ACMTF was compared to PCA, a widely used method to decompose molecular formula tables. ${ }^{15}$ The first significant difference between PCA and ACMTF is the ability to impose non-negativity constraints in ACMTF. Our analysis indicated that loadings and scores of PC1-PC6 in PCA were both positive and negative (Figure S10), while ACMTF component loadings were exclusively positive (Figure 2, panel C). In ACMTF, component loadings and scores therefore directly correspond to analytical signals, while in the case of PCA, the interpretation of components is less intuitive since a combination of negative and positive scores and loadings must be considered. The option to constrain models to nonnegative loadings and scores in ACMTF represents an improvement in the characterization of complex mixtures such as DOM with mass spectrometry.

Compared to the strict orthogonality of components in PCA, ACMTF allows some degree of similarity between molecular formula loadings. A congruence analysis between all unique combinations of the six ACMTF components showed that ACMTF loadings of molecular formula components were 

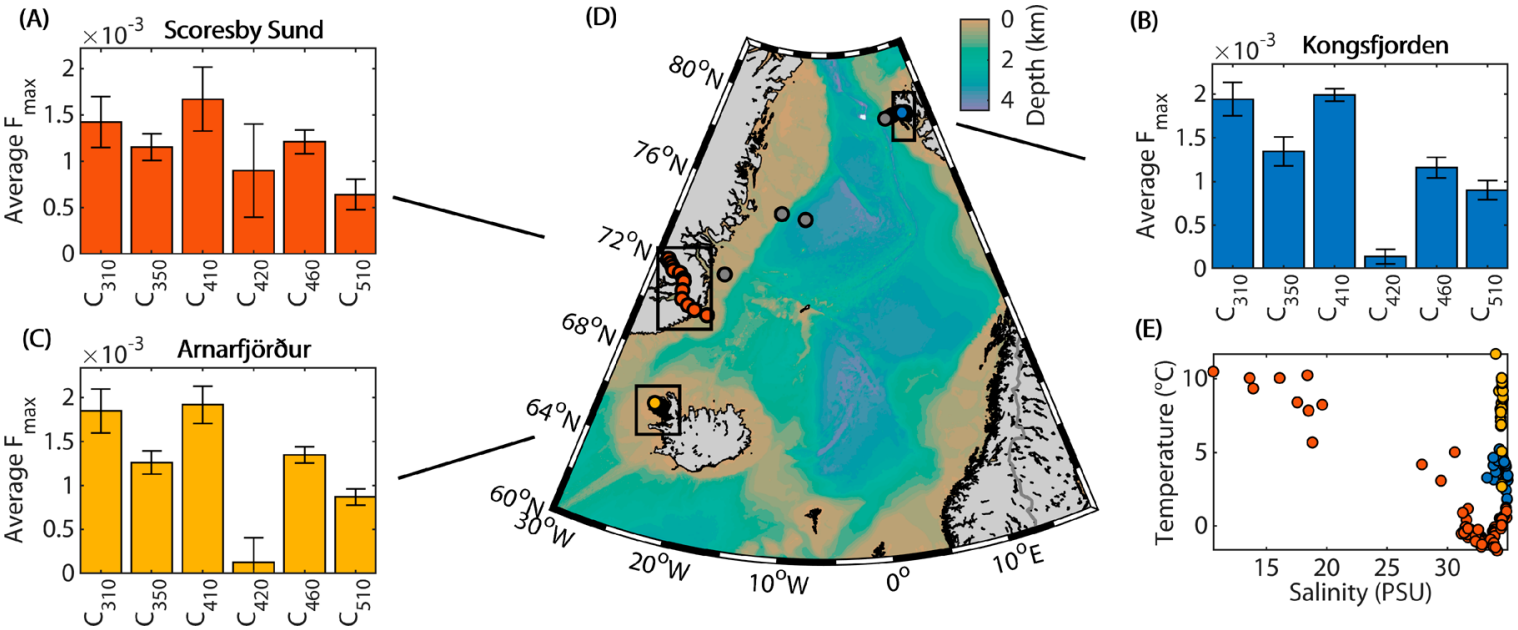

Figure 4. Average distribution of ACTMF components across three Arctic fjords. Average scores are shown in A-C and colored according to the respective fjord in the map (D). Error bars indicate the standard deviation of the mean. For oceanographic context, a temperature-salinity plot is shown in (E). Sampling stations outside of the fjords are marked in gray and not shown in other plots.

autocorrelated to some degree (between 0.21 and 0.85 , on a scale from zero to one, Figure S11). The similarity between ACMTF components highlights that environmental processes or independent chemical fractions may overlap in their spectral properties. Models with strictly orthogonal components (such as PCA) would ultimately be unable to recover these spectra. Together, our comparison indicated that ACMTF provides more chemically intuitive results, making it a more appropriate model for the decomposition of molecular formula matrices of DOM. However, it is important to stress that ACMTF models are primarily driven by the variability of the tensor (fluorescence EEMs). The description of variability beyond EEMs depends on fitting unshared components that can describe variability independent of fluorescence. Future efforts should also include the comparison of components derived from data fusion using ACMTF and those derived from factorizations based solely on molecular formulas to investigate how data fusion models relate to models describing only molecular formulas. However, this is pending the validation of non-negative matrix factorizations applicable to molecular formula data sets of DOM, and a comparison is thus not yet possible.

Since ACMTF is based on PARAFAC, there should be a basic agreement between loadings and scores of a PARAFAC model fit to the EEM data exclusively, and the ACMTF model describing both EEMs and molecular formulas. A detailed comparison between ACMTF and PARAFAC is given in the SI (SI S2, Figure S8). In short, ACMTF loadings were highly congruent with corresponding PARAFAC components, while the scores of some components $\left(\mathrm{C}_{420}, \mathrm{C}_{460}\right)$ diverged from the PARAFAC solution despite clearly showing a positive correlation. These discrepancies are most likely attributable to the occurrence of mass spectrometry-specific disturbances. Among other possibilities, matrix effects in ESI-MS affecting certain groups of samples could have caused deviations in component scores of shared components. Despite this, the simultaneous factorization of fluorescence EEMs and the molecular formula table resulted in a model that generally agreed with PARAFAC.

Finally, we compared associations between fluorescence EEMs and molecular formulas identified by ACMTF with the correlations identified by the post hoc Pearson rank correlation of PARAFAC $F_{\max }$ values and molecular formula abundances (Figure 2, panel D). Although, there was a degree of overlap between associations identified by each approach (Figure 2, panel C, D), there was also substantial disagreement. The correlation-weighted mass spectra (showing correlation coefficient in place of abundances) were compared to ACMTF component mass spectra, using the Tucker congruence coefficient. For all comparisons, TCCs were smaller than 0.45 , indicating poor agreement. In extreme cases, such as $C_{310}$, $\mathrm{C}_{350}$, and $\mathrm{C}_{460}$, correlations were found to be inverse in areas of the van Krevelen space that showed relatively low but positive loadings in corresponding ACMTF components. $\mathrm{C}_{510}$ was inversely correlated with highly oxygenated, highly unsaturated molecular formulas in the rank analysis, while ACMTF indicated a strong positive correlation in that compositional space.

Comparisons between data fusion and rank correlation are hindered by some key differences between the correlation and data fusion approaches. Rank correlations in their simplest interpretation provide a binary indication of direct or inverse association between fluorescence and molecular formulas. This approach does not decompose a multivariate signal and often returns multiple possible correlations between variables. This leaves users to decide which of the significant correlations are valid, and which can (or should) be ignored. On the other hand, data fusion readily addresses this issue by dissecting a particular molecular formula abundance into multiple components. This multivariate decomposition represents a more objective approach that is far less vulnerable to false positive errors and thus provides more robust estimates of the molecular fingerprint of fluorescent DOM.

Biogeochemical Sources, Chemical Fractions, or Diagenetic State? DOM is a highly complex mixture of organic compounds with contrasting chemical properties, varying biogeochemical sources and sinks, and different degradation potential. It is striking that more than $99 \%$ of the modeled mass spectral variability ( $\sim 38 \%$ of the mass spectra) was described by only six statistical components. This represents a significant reduction in complexity that facilitates a far easier interpretation of DOM data sets. However, the partitioning of DOM fluorescence and molecular formulas into statistical components poses the question: Do ACMTF 

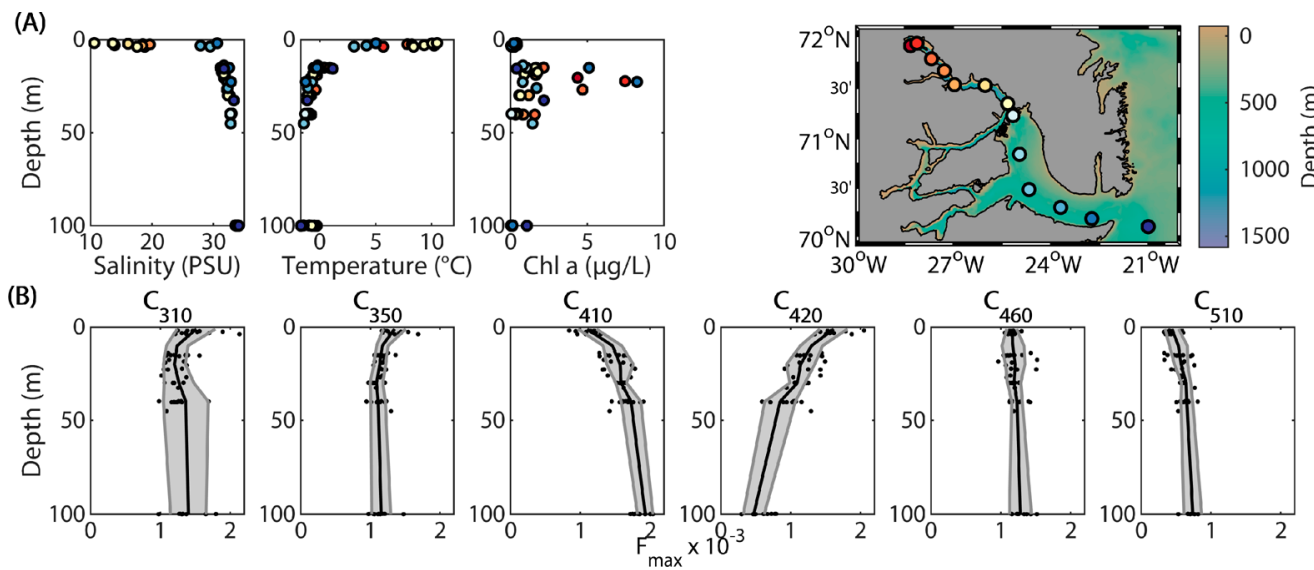

Figure 5. Depth variation of physical and environmental parameters in Scoresby Sound. Panel A: Salinity, temperature, and chlorophyll a, along with a station map. Panel B: Depth profiles of the six ACMTF component $F_{\max }$ values (surface to $100 \mathrm{~m}$, the actual water column depth was greater). Dots represent $F_{\max }$ values, black lines the binned average along with the standard deviation of the mean (gray).

components indicate biogeochemical source materials (e.g., terrestrial substances), reflect its diagenetic state (e.g., recalcitrant material), or represent distinct chemical fractions (e.g., high molecular weight DOM)?

To assign an interpretation to the six ACMTF components, across-fjord patterns (Figure 4) and depth-dependent trends in Scoresby Sound (Figure 5) were investigated while bearing in mind the molecular fingerprint of respective components (Figure 2C). The most distinct oceanographic pattern was observed for $\mathrm{C}_{420}$, which was most prevalent in Scoresby Sound (Figure 4). In Scoresby Sound, $F_{\max }$ values of $\mathrm{C}_{420}$ also exhibited a distinct surface maximum, followed by a decrease in its relative abundance with depth, and a subsurface peak at all stations in the polar waters $(S<34, T<0)$ was observed (Figure 5). Since Scoresby Sound receives terrestrial material from the EGC, terrestrial material is most likely a major source of this component. However, because a recent study identified a component highly similar to $\mathrm{C}_{420}$ as ubiquitous across a wide range of environments, ${ }^{40} \mathrm{C}_{420}$ is likely not a highly selective proxy for terrestrial material but rather represents a fluorescence fraction that is particularly abundant in terrestrially influenced waters. Interestingly, $\mathrm{C}_{420}$ had the lowest average molecular weight, was strongly associated with the most oxygenated, unsaturated molecular formulas, and showed the highest chemodiversity, suggesting that terrestrially derived substances represent a distinguishable chemical fraction. The identification of this terrestrially dominated component provides targets for further experiments, for example, via MS/MS to explore molecular structures.

Components fluorescing in the spectral range of $\mathrm{C}_{350}$ are commonly termed "protein-like"; a term which is derived from the apparent spectral similarity with amino acid fluorescence that has been shown to correlate with amino acid concentration. ${ }^{41}$ The presence of a UV-A fluorescence signature has often been used as a proxy for surface water biological activity, and recent studies have demonstrated that such processes impact DOM mass spectra with high selectivity. ${ }^{42}$ In agreement with these findings, ACMTF indicated a low chemodiversity of the molecular fingerprint associated with $\mathrm{C}_{350}$, which can be interpreted as representing fresh organic material closely linked to planktonic productivity in the surface layer (Figure 5). Similar to $\mathrm{C}_{350}, \mathrm{C}_{310}$ also exhibited a subsurface decrease in Scoresby Sound $(10-30 \mathrm{~m}$ ) but slightly increased again at $45 \mathrm{~m}$. The higher chemodiversity of $\mathrm{C}_{310}$ compared to $\mathrm{C}_{350}$ suggests that $\mathrm{C}_{310}$ may encompass degradation products related to lateral terrestrial inputs, plankton productivity, or (photo)degradation in the surface layer. A more constrained assignment is not possible at present and would require further experiments.

For the remaining components, across-fjord differences were subtle, while depth profiles differed between components. The composition in Arnarfjörđur and Kongsfjorden was relatively stable with depth (Figures S12 and S13), whereas distinct changes were observed in Scoresby Sound (Figure 5). $\mathrm{C}_{410}$ and $\mathrm{C}_{510}$ increased with depth, suggesting that this moderately complex fingerprint was possibly impacted by the processing of sinking organic matter. In contrast, $\mathrm{C}_{460}$ was invariant with water depth in all three systems, which suggests it may represent a recalcitrant component of high molecular complexity (Figure 5, also see Figure S12-13). These systematic trends provide further confidence in the components identified by this data fusion approach.

Challenges and Future Directions. Multivariate data fusion simultaneously decomposes multiple analytical data sets and provides a tool to link heterogeneous data sets, such as fluorescence spectroscopy and mass spectrometry. The methodological similarities with PARAFAC provide the opportunity to integrate data fusion algorithms into popular existing software toolboxes (such as $\mathrm{drEEM}$ ), as well as related public databases (such as OpenFluor). With the continuous development of multicore processors and improvements in modeling algorithms, the computational expense of data fusion will greatly decrease in the future, making it widely applicable for the scientific community. While multivariate data fusion may not be able to identify the chemical compounds responsible for the optical properties of complex environmental DOM data sets, it greatly improves the chemical interpretability of fluorescence data sets and offers potential for future developments. With data fusion as a central interface, future studies will be able to leverage the superior analytical depth of mass spectrometry while utilizing the spatiotemporal resolution of ultraviolet-visible spectroscopy.

Assigning an interpretation to ACMTF components is confounded by the increased information content of components, particularly the high complexity of molecular fingerprints. Whereas fluorescence components are continuous, and their chemical interpretation may generally be assessed by comparison to pure fluorophore spectra, the 
complex and discontinuous nature of component mass spectra constitutes a significant challenge in this regard. In light of their generally high diversity, it appears reasonable to assume that ACMTF component mass spectra represent multiple, currently unresolvable chemical fractions. The ability to further separate chemical fractions may be improved by integrating additional DOM analyses, such as absorbance or ${ }^{13} \mathrm{C}$ or ${ }^{1} \mathrm{H}$ nuclear magnetic resonance (NMR) spectroscopy. ACMTF is theoretically able to link one trilinear data set (fluorescence EEMs) with multiple matrices (such as ${ }^{13} \mathrm{C}$ and ${ }^{1} \mathrm{H}$ NMR). However, ACMTF requires that one of the data structures be trilinear. The fusion of bilinear data sets, such as FT-ICR-MS and ${ }^{13} \mathrm{C} N M R$, requires other data fusion strategies. ${ }^{43}$

There are a number of methodological challenges that remain to be solved in regard to the applicability of ACMTF to DOM data sets. For example, it is very likely that FDOM EEMs and FT-ICR-MS formula matrices do not share the same number of underlying components. While ACMTF is able to address this disparity between two data sets with dataset- and component-specific weights, it is currently unknown how well this approach can cope with the potentially large discrepancy between the number of components in fluorescence vs molecular formula data sets of DOM. An expanded analysis of this is warranted, and this topic needs consideration in future efforts. Furthermore, our study put focus on the $\mathbf{5 2 7}$ most conserved molecular formulas since the primary goal was to identify the molecular fingerprint of quasi-ubiquitous fluorescence signals. Relaxing the data preprocessing criteria by including more unique (uncommon) molecular formulas would be desirable from a biogeochemical point of view. However, this presents a challenge for model validation and considerably increases computation time. In order to include sparsely observed molecular formulas, further developments of the modeling approach are necessary.

The scientific community utilizing FDOM EEMs has converged toward a standard methodology for measuring samples and analyzing resulting data sets with PARAFAC, achieved through a substantial number of efforts including interlaboratory comparisons. ${ }^{12,23,44}$ This was driven mostly by the fact that multivariate analysis demands a stringent and standardized sample and data processing routine, to provide globally consistent data. The measurement and data analysis of FT-ICR-MS mass spectra is in the process of being standardized, and this is essential if advanced data analysis techniques are to be employed. ${ }^{45}$ For the time being, however, different guidelines for formula assignment, peak identification, and signal normalization exist across the community, and no central, open database has been developed to our knowledge. If data fusion should become a viable tool for community-wide DOM characterization, these discrepancies must be addressed to provide reproducible results that enable replication.

Key differences between fluorescence spectroscopy and FTICR-MS currently complicate joint description of fluorescence and ESI-MS data sets. Fluorescence quantum yields and ESI efficiencies of analytes are unknown; therefore, peak intensities and proportions between peaks reflect a combination of actual concentrations and differences in fluorescence and ionization efficiencies, respectively. However, ESI-MS peak intensities differ from fluorescence, because carbon concentrations of samples are typically adjusted before injection, and signals are normalized to the sum of peaks (or the highest peak) during postprocessing. These steps are designed to improve data robustness, but peak abundances subsequently depend on (1) ionization efficiencies and (2) the abundance of the remaining peaks in relation to the total carbon concentration. To ensure the compatibility of the two data sets during data fusion, fluorescence signals must currently be normalized to the total fluorescence per sample. As a result, ACMTF scores represent proportions. Obtaining more quantitative ACMTF component scores hinges on developing approaches that yield robust mass spectra without a carbon- and peak-normalization step.

Beyond issues related to sample and data treatment, practical issues related to the ion source may further compromise the ability to quantify analytes with ESI-MS. The ionization of DOM constituents with ESI is inherently selective, and matrix effects introduce artifacts. ${ }^{3,46}$ However, the extent to which matrix effects impact DOM mass spectra remains poorly quantified as the molecular structures of DOM remain largely uncharacterized. Nonetheless, matrix effects have been documented for isolated marine metabolites. ${ }^{47}$ The application of models assuming linear relationships between analyte concentration and detector signal thus requires careful investigation. Here, we observed that the majority of the 527 modeled formulas could be described using a model that assumes a linear relationship between fluorescence signals and molecular formula abundances. The reproducibility of our efforts should be investigated in future studies carried out across a wide variety of aquatic environments.

\section{ASSOCIATED CONTENT}

\section{Supporting Information}

The Supporting Information is available free of charge on the ACS Publications website at DOI: 10.1021/acs.analchem.8b02863.

Supplementary methods (S1), supplementary results containing information on model validation (S2), 13 supplementary figures (S3), and two supplementary tables (S4) (PDF)

\section{AUTHOR INFORMATION}

\section{Corresponding Author}

*E-mail: wuensch@chalmers.se. Present address: Sven Hultins Gata 6, 41296 Gothenburg, Sweden.

ORCID $\odot$

Urban J. Wünsch: 0000-0001-6972-6932

Kathleen R. Murphy: 0000-0001-5715-3604

Notes

The authors declare no competing financial interest.

\section{ACKNOWLEDGMENTS}

This study was in part funded by the Danish Council for Independent Research-Natural Sciences Grant DFF-132300336 and Nordic5Tech collaborative funding (Technical University of Denmark). K.R.M. and U.J.W. acknowledge funding from the Swedish Research Council (FORMAS 201700743).We greatly appreciate the constructive comments by four anonymous reviewers and the editor Professor Dovichi, which helped to improve this manuscript. We thank the captain and crew of R/V Maria S. Merian for their support during cruise MSM56. Mourad Harir (Helmholtz Zentrum München), Oliver J. Lechtenfeld (Helmholtz-Zentrum für Umweltforschung UFZ), Claudia Burau, and Jana K. Geuer (both Alfred Wegener Institute for Polar and Marine Research) are acknowledged for their help during sampling, 
extract preparations, and FT-ICR-MS measurements. U.J.W. thanks Rasmus Bro (Copenhagen University) for helpful discussions on the ACMTF model. E.A. and U.J.W. thank Michael Saunders (Stanford University) for kindly providing access to the SNOPT toolbox.

\section{REFERENCES}

(1) Buchan, A.; LeCleir, G. R.; Gulvik, C. A.; González, J. M. Nat. Rev. Microbiol. 2014, 12 (10), 686-698.

(2) Mopper, K.; Kieber, D. J.; Stubbins, A. Marine Photochemistry of Organic Matter: Processes and Impacts. Processes and Impacts, second ed.; Elsevier Inc., 2014.

(3) Repeta, D. J. Chemical Characterization and Cycling of Dissolved Organic Matter. In Biogeochemistry of Marine Dissolved Organic Matter; Elsevier Inc., 2015; pp 21-63.

(4) Carlson, C. A.; Hansell, D. A. DOM Sources, Sinks, Reactivity, and Budgets. In Biogeochemistry of Marine Dissolved Organic Matter, second edition; Elsevier Inc., 2014; pp 65-126.

(5) Koch, B. P.; Witt, M.; Engbrodt, R.; Dittmar, T.; Kattner, G. Geochim. Cosmochim. Acta 2005, 69 (13), 3299-3308.

(6) Hertkorn, N.; Frommberger, M.; Witt, M.; Koch, B. P.; SchmittKopplin, P.; Perdue, E. M. Anal. Chem. 2008, 80 (23), 8908-8919.

(7) Mopper, K.; Stubbins, A.; Ritchie, J. D.; Bialk, H. M.; Hatcher, P. G. Chem. Rev. 2007, 107 (2), 419-442.

(8) Fellman, J. B.; Hood, E.; Spencer, R. G. M. Limnol. Oceanogr. 2010, 55 (6), 2452-2462.

(9) Coble, P. G. Mar. Chem. 1996, 51 (4), 325-346.

(10) Stedmon, C. A.; Nelson, N. B. The Optical Properties of DOM in the Ocean. In Biogeochemistry of Marine Dissolved Organic Matter; Hansell, D. A., Carlson, C. A., Eds.; Elsevier Inc.: San Diego, 2015; pp 481-508.

(11) Ferdinand, O. D.; Friedrichs, A.; Miranda, M. L.; Voß, D.; Zielinski, O.. Next Generation Fluorescence Sensor with Multiple Excitation and Emission Wavelengths - NeXOS. In OCEANS 2017 Aberdeen; IEEE: Aberdeen, 2017; pp 1-6.

(12) Murphy, K. R.; Stedmon, C. A.; Graeber, D.; Bro, R. Anal. Methods 2013, 5 (23), 6557-6566.

(13) Rosario-Ortiz, F. L.; Korak, J. A. Environ. Sci. Technol. 2017, 51 (2), 759-761.

(14) Stenson, A. C.; Marshall, A. G.; Cooper, W. T. Anal. Chem. 2003, 75 (6), 1275-1284.

(15) Sleighter, R. L.; Liu, Z.; Xue, J.; Hatcher, P. G. Environ. Sci. Technol. 2010, 44 (19), 7576-7582.

(16) Acar, E.; Gürdeniz, G.; Savorani, F.; Hansen, L.; Olsen, A.; Tjønneland, A.; Dragsted, L. O.; Bro, R. J. Proteome Res. 2017, 16 (7), 2435-2444.

(17) Stubbins, A.; Lapierre, J.-F.; Berggren, M.; Prairie, Y. T.; Dittmar, T.; del Giorgio, P. A. Environ. Sci. Technol. 2014, 48, 1059810606.

(18) Herzsprung, P.; Von Tümpling, W.; Hertkorn, N.; Harir, M.; Büttner, O.; Bravidor, J.; Friese, K.; Schmitt-Kopplin, P. Environ. Sci. Technol. 2012, 46 (10), 5511-5518.

(19) Acar, E.; Papalexakis, E. E.; Gürdeniz, G.; Rasmussen, M. A.; Lawaetz, A. J.; Nilsson, M.; Bro, R. BMC Bioinf. 2014, 15 (1), 239.

(20) Acar, E.; Bro, R.; Smilde, A. K. Proc. IEEE 2015, 103 (9), $1602-1620$.

(21) Acar, E.; Levin-Schwartz, Y.; Calhoun, V. D.; Adal1, T. TensorBased Fusion of EEG and FMRI to Understand Neurological Changes in Schizophrenia. (2017) 2017 IEEE International Symposium on Circuits and Systems (ISCAS); IEEE: Baltimore, MD, p 1 .

(22) Dittmar, T.; Koch, B. P.; Hertkorn, N.; Kattner, G. Limnol. Oceanogr.: Methods 2008, 6, 230-235.

(23) Kothawala, D. N.; Murphy, K. R.; Stedmon, C. A.; Weyhenmeyer, G. A.; Tranvik, L. J. Limnol. Oceanogr.: Methods 2013, 11 (DEC), 616-630.
(24) Flerus, R.; Lechtenfeld, O. J.; Koch, B. P.; McCallister, S. L.; Schmitt-Kopplin, P.; Benner, R; Kaiser, K.; Kattner, G. Biogeosciences 2012, 9 (6), 1935-1955.

(25) Lechtenfeld, O. J.; Koch, B. P.; Gašparović, B.; Frka, S.; Witt, M.; Kattner, G. Mar. Chem. 2013, 150, 25-38.

(26) Acar, E.; Kolda, T. G.; Dunlavy, D. M. All-at-Once Optimization for Coupled Matrix and Tensor Factorizations. (2011) In KDD Workshop on Mining and Learning with Graphs. https://arxiv.org/abs/1105.3422.

(27) Bader, B. W.; Kolda, T. G. (2012) MATLAB Tensor Toolbox. Available online at https://www.tensortoolbox.org.

(28) Gill, P. E.; Murray, W.; Saunders, M. A. SIAM Rev. 2005, 47 (1), 99-131.

(29) Kellerman, A. M.; Dittmar, T.; Kothawala, D. N.; Tranvik, L. J. Nat. Commun. 2014, 5 (1), 1-8.

(30) Chao, A.; Shen, T.-J Environ. Ecol. Stat. 2003, 10, 429-443.

(31) Wünsch, U. J.; Geuer, J. K.; Lechtenfeld, O. J.; Koch, B. P.; Murphy, K. R.; Stedmon, C. A. Mar. Chem. 2018, DOI: 10.1016/ j.marchem.2018.08.010.

(32) Lorenzo-Seva, U.; ten Berge, J. M. F. Methodology 2006, 2 (2), 57-64.

(33) Holm, S. A. Scand. J. Stat. 1979, 6 (2), 65-70.

(34) Murphy, K. R.; Stedmon, C. A.; Wenig, P.; Bro, R. Anal. Methods 2014, 6 (3), 658-661.

(35) Walker, S. A.; Amon, R. M. W.; Stedmon, C. A.; Duan, S.; Louchouarn, P. J. Geophys. Res. 2009, 114 (4), 1-12.

(36) Shutova, Y.; Baker, A.; Bridgeman, J.; Henderson, R. K. Water Res. 2014, 54, 159-169.

(37) Graeber, D.; Gelbrecht, J.; Pusch, M. T.; Anlanger, C.; von Schiller, D. Sci. Total Environ. 2012, 438, 435-446.

(38) Yamashita, Y.; Kloeppel, B. D.; Knoepp, J.; Zausen, G. L.; Jaffé, R. Ecosystems 2011, 14 (7), 1110-1122.

(39) Stedmon, C. A.; Markager, S.; Tranvik, L. J.; Kronberg, L.; Slätis, T.; Martinsen, W. Mar. Chem. 2007, 104 (3-4), 227-240.

(40) Murphy, K. R.; Timko, S. A.; Gonsior, M.; Powers, L. C.; Wünsch, U. J.; Stedmon, C. A. Environ. Sci. Technol. 2018, 52 (19), $11243-11250$.

(41) Yamashita, Y.; Tanoue, E. Mar. Chem. 2003, 82 (3-4), 255271.

(42) Reader, H. E.; Stedmon, C. A.; Nielsen, N. J.; Kritzberg, E. S. Front. Mar. Sci. 2015, 2, 1-10.

(43) Smilde, A. K.; Måge, I.; Næs, T.; Hankemeier, T.; Lips, M. A.; Kiers, H. A. L.; Acar, E.; Bro, R. J. Chemom. 2017, 31 (7), e2900.

(44) Murphy, K. R.; Butler, K. D.; Spencer, R. G. M.; Stedmon, C. A.; Boehme, J. R.; Aiken, G. R. Environ. Sci. Technol. 2010, 44 (24), 9405-9412.

(45) Leefmann, T.; Frickenhaus, S.; Koch, B. P. Rapid Commun. Mass Spectrom. 2018, DOI: $10.1002 / \mathrm{rcm} .8315$.

(46) Taylor, P. J. Clin. Biochem. 2005, 38 (4), 328-334.

(47) Johnson, W. M.; Kido Soule, M. C.; Kujawinski, E. B. Limnol. Oceanogr.: Methods 2017, 15 (4), 417-428. 